\title{
PARÂMETROS r E R OBTIDOS DE PROGRAMA INTERLABORATORIAL - COMO USÁ-LOS
}

\author{
Queenie Siu Hang Chui*, Cleide Bassani de Barros e Talita Daiane da Silva \\ Laboratório de Metrologia Química, Universidade São Francisco, Rua Alexandre Rodrigues Barbosa, 45, 13250-901 Itatiba - SP, \\ Brasil
}

Recebido em 20/10/08; aceito em 13/4/09; publicado na web em 17/9/09

\begin{abstract}
REPEATABILITY ( $\mathrm{r}$ ) AND REPRODUCIBILITY (R) INDEXES FROM INTERLABORATORIAL PROGRAM - HOW TO USE THEM. Intralaboratorial and interlaboratorial variabilities can be obtained by conducting an specially designed interlaboratory program. Using the analysis of variance technique one can calculate the $\mathrm{r}$ (repeatability) and $\mathrm{R}$ (reproducibility) indexes that can help participant laboratories to monitor their routine quality control procedures. An example is described using data obtained from an interlaboratory program where twelve laboratories determined total iron content in silicon metal sample by using flame atomic absorption spectrometry.
\end{abstract}

Keywords: repeatability; reproducibility; inter and intralaboratorial variance.

\section{INTRODUÇÃO}

Nos últimos anos, os laboratórios analíticos vêm usando comparações interlaboratoriais para o controle externo da qualidade laboratorial. Além do seu papel como uma ferramenta de controle de qualidade externo, resultados de programas interlaboratoriais, ou amostras, podem ser usados como uma alternativa para alcançar alguns requisitos de garantia da qualidade, tais como precisão analítica, avaliação da incerteza e controle interno da qualidade. Este uso adicional do programa interlaboratorial pode ajudar os laboratórios participantes a reduzirem o impacto financeiro do seu processo de qualidade assegurada. ${ }^{1}$

Para obter dados válidos e confiáveis, o procedimento analítico deve estar sob controle estatístico e ser executado sob um sistema de gestão da qualidade bem estabelecido, sendo os programas interlaboratoriais um componente inerente. A participação regular em programas interlaboratoriais, quando disponível, é recomendada pela NBR ISO/IEC $17025,{ }^{2}$ a fim de atender o requisito 5.9, que diz: "O laboratório deve ter procedimentos de controle da qualidade para monitorar a validade dos ensaios". E, mais adiante: "O monitoramento deve ser planejado e analisado criticamente e pode incluir, mas não estar limitado ao seguinte: a) uso regular de materiais de referência certificados; b) participação em programas de comparação interlaboratorial ou de ensaios de proficiência”.

Em situação ideal, o desempenho do laboratório em um programa interlaboratorial, quando organizado adequadamente de acordo com os princípios do guia NBR ISO/IEC $43-1,{ }^{3}$ demonstra sua competência. Na prática, observa-se que os laboratórios que participam de comparações interlaboratoriais ainda não aproveitam completamente os dados gerados pelo programa e seus benefícios.

O benefício mais importante a ser usufruído pelos laboratórios participantes é ter meios de se comparar com laboratórios similares e identificar problemas que os laboratórios, quando trabalhando isolados, não poderiam descobrir. ${ }^{4}$ Outra possibilidade é monitorar os resultados dos laboratórios participantes obtidos por métodos diferentes, facilitando a comparação de desempenho de métodos em materiais idênticos e possibilitando verificar a influência de diversos fatores responsáveis pela variabilidade em resultados de análises.

\footnotetext{
*e-mail: queenie.hang@saofrancisco.edu.br
}

\section{PROGRAMAS INTERLABORATORIAIS}

A definição dada pela NBR ISO/IEC 43-1 para Programa ou Comparação Interlaboratorial é bastante abrangente: "organização, desempenho e avaliação de ensaios nos mesmos itens ou em itens de ensaios similares por dois ou mais laboratórios de acordo com condições pré-determinadas". ${ }^{3}$

Programa interlaboratorial é uma ferramenta utilizada para diferentes finalidades, tais como avaliar a competência técnica de laboratórios (Ensaios de Proficiência); para a compatibilização de resultados de diversos laboratórios, quando não existem valores de referência a serem atribuídos à propriedade desejada; para a determinação de parâmetros de precisão de metodologias e, para certificação de materiais de referência. ${ }^{5}$

Por meio de um programa interlaboratorial específico, é possível a determinação de parâmetros de precisão para métodos de ensaios. Resultados de ensaios, mesmo obtidos com métodos normalizados, apresentam variabilidades. Na interpretação de resultados de ensaios, estas variabilidades devem ser consideradas.

Precisão, segundo ISO/IEC 5725-1, ${ }^{6}$ é "definida como o grau de concordância entre resultados de medições independentes, em torno de um valor central, efetuadas várias vezes em uma amostra homogênea, sob condições experimentais pré-estabelecidas". Depende somente da distribuição de erros aleatórios e não está vinculada ao "valor verdadeiro" ou valor designado. ${ }^{7}$ Desvio padrão é a expressão quantitativa da precisão. Medidas quantitativas da precisão dependem criticamente das condições pré-estabelecidas.

Alguns documentos existentes, como o DOQ-CGCGRE-008INMETRO $^{8}$ e a norma brasileira ABNT NBR $14597,{ }^{9}$ abordam a determinação dos limites de precisão, os chamados limites de repetitividade e reprodutibilidade, em ambientes intralaboratoriais, orientando para sua abordagem no contexto da validação de métodos analíticos.

O presente trabalho faz uma nova abordagem para a obtenção de parâmetros de precisão de método de ensaio, em que se envolvem vários laboratórios participando de programa colaborativo interlaboratorial. A vantagem para esses laboratórios é poder usufruir de uma ferramenta válida para o monitoramento dos processos críticos de rotina, representando um meio externo para o controle da qualidade analítica.

Em programas interlaboratoriais, os parâmetros de precisão são reportados pelo desvio padrão obtido em condições de repetitividade $\left(\mathrm{s}_{\mathrm{r}}\right)$ e desvio padrão obtido em condições de reprodutibilidade $\left(\mathrm{s}_{\mathrm{R}}\right)$. 
A partir destes parâmetros obtêm-se os denominados índices de repetitividade - repê (r) e reprodutibilidade - Reprô (R) calculados para descrever as variabilidades de um método de ensaio, a partir das variabilidades intrínsecas e extrínsecas de todos os laboratórios..$^{10,11}$ A variabilidade intrínseca corresponde à repetitividade da medição, e a variabilidade extrínseca, associada à reprodutibilidade, engloba contribuições das variabilidades internas, do erro entre laboratórios e do efeito da interação, se houver.

Os índices propostos são calculados a partir da decomposição da variância global de acordo com a causa ou fator causador. Os índices dependerão do nível de confiança desejado, a ser definido, usualmente adotado como $95 \%$. Outros fatores importantes são o número de laboratórios e o número de replicatas que deve ser explicitado ao ser indicado o valor dos índices.

\section{ANÁLISE DE VARIÂNCIA - FATOR ÚNICO}

É a técnica estatística mais simples de análise de variância. É usada para testar diferenças significativas entre médias de vários conjuntos de resultados e separar e estimar as variabilidades associadas com fontes definidas. ${ }^{12,13}$

Em programas interlaboratoriais quando vários laboratórios analisam uma amostra, são consideradas a variância residual relacionada à variância da repetitividade e a variância entre laboratórios, que é a combinação das variâncias entre laboratórios, interação amostra/ laboratório (se houver) e as variâncias dentro de laboratórios.

Para seu uso adequado existem duas premissas: 1) para cada nível de concentração as médias obtidas em diferentes laboratórios são normalmente distribuídas; esta hipótese pode ser aceita sem muitas restrições e os desvios da normalidade não são muito críticos, ${ }^{10}$ especialmente com número grande de observações. 2) para cada concentração, as variâncias em cada grupo ou laboratório são as mesmas, ou seja, existe homocedasticidade das variâncias. ${ }^{11}$ Assim, é recomendável aplicar um teste estatístico para verificar se existe igualdade de variâncias.

Vários são os testes citados em literatura, ${ }^{11-14}$ porém os mais conhecidos são o de Bartlett e o de Cochran. O primeiro não pode ser aplicado quando uma das variâncias, em um conjunto, for zero, o que pode acontecer facilmente como consequência de arredondamentos de resultados de ensaios, cujos valores são bem baixos.

Seguindo as instruções da norma ISO 5725-2, ${ }^{15}$ que instrui sobre os procedimentos a serem adotados em programas interlaboratoriais, será utilizado o teste de Cochran, que verifica as variâncias, seguido do teste de Grubbs, que verifica a compatibilidade de médias.

Com a decomposição das variâncias, os valores dos quadrados médios indicam os componentes de variância atribuíveis às diferentes fontes de variação: quadrados médios dentro de níveis ou grupos $Q M_{\text {dentro }}$, ou seja, a variância residual; e quadrados médios entre níveis ou grupos - $Q M_{\text {entre }}$, que representa a combinação das variâncias entre laboratórios, interação amostra/laboratório (se houver) e as variâncias dentro de laboratório. ${ }^{12,13}$

A estimação dos componentes de variância, com base nos resultados da análise de variância, é obtida como segue: ${ }^{16}$

$s_{\text {dentro }}^{2}=Q M_{\text {dentro }}$

$s_{\text {entre }}^{2}=\frac{Q M_{\text {entre }}-Q M_{\text {dentro }}}{n}$

A montagem formal da resolução do problema da análise de variância relativa ao modelo para fator único é corriqueiramente apresentada em literatura e, por esse motivo, não será aqui aprofundada. ${ }^{11-13,16}$ Na prática, para obter o processamento dos dados, usa-se a planilha Excel ou outro software para o cálculo dos quadrados médios. Com este procedimento, obtêm-se os valores das variâncias em condições de repetitividade $\left(s_{r}^{2}\right)$ e de reprodutibilidade $\left(s^{2}\right)$, a saber:

$$
\begin{aligned}
& \text { variância da repetitividade } \rightarrow s_{r}^{2}=s_{\text {dentro }}^{2} \\
& \text { variância da reprodutibilidade } \rightarrow s^{2}{ }_{R}=s_{\text {entre }}{ }^{2}+s^{2}{ }_{\text {dentro }}
\end{aligned}
$$

\section{ÍNDICES DE REPETITIVIDADE E DE REPRODUTIBILIDADE}

Os índices $\mathrm{r}$ e R são obtidos associando-se às variâncias coeficientes que levam em consideração o grau de confiança desejado e a forma da distribuição. Para uma distribuição normal no nível de $95 \%$ de confiança, o valor é igual a $f \cdot \sqrt{2}=1,96 \sqrt{2}=2,77 \cong 2,8$ válido para a diferença máxima permitida entre dois resultados.

A rigor, segundo a norma ISO 5725-6 os valores dos fatores $f(n)$ estão em função do valor de $(n)$, para um nível de confiança relacionado. ${ }^{17}$ Conforme a Tabela 1 , para $n=3, f(n)=3,3$ e para $n=4, f(n)$ $=3,6$ e assim por diante.

Tabela 1. Fator da diferença crítica $-f_{(n)}(95 \%$ de confiança) em função do número " $\mathrm{n}$ " de replicatas

\begin{tabular}{cccccccccccc}
\hline $\mathrm{n}$ & 2 & 3 & 4 & 5 & 6 & 7 & 8 & 9 & 10 & 11 & 12 \\
\hline$f_{(n)}$ & 2,8 & 3,3 & 3,6 & 3,9 & 4,0 & 4,2 & 4,3 & 4,4 & 4,5 & 4,6 & 4,6 \\
\hline
\end{tabular}

Fonte: ref. 17

Na prática, usa-se o valor 2,8 independente do tamanho do estudo, para verificar a diferença de dois resultados, prática normalmente adotada em trabalhos de rotina em laboratórios. Assim,

$\mathrm{r}($ repê $)=f(n) s_{r}=2,8 s_{\text {dentro }}$

$\mathrm{R}($ Reprô $)=f(n) s_{R}=2,8\left(s_{\text {dentro }}^{2}+s_{\text {entre }}^{2}\right)^{1 / 2}$

Compara-se a diferença entre dois (ou mais) resultados intralaboratoriais com o valor de (r). Quando a diferença absoluta entre dois resultados não excede o valor da repetitividade (r), então os dois resultados são aceitáveis e pode-se calcular a média aritmética. Quando a diferença absoluta entre os dois resultados excede o valor da repetitividade (r), deve-se obter mais um resultado (caso típico de ensaios complexos ou demorados) ou mais dois resultados (caso típico de ensaios fáceis ou rápidos). Se a amplitude de 3 resultados não excede o valor $f_{(n)} . s_{r}$, calcula-se a média aritmética dos 3 resultados; caso contrário, considera-se a mediana dos 3 resultados ou obtém-se mais 1 resultado. Da mesma forma, se a amplitude dos 4 resultados não excede o valor $f_{(n)} . s_{r}$, calcula-se a média aritmética dos 4 resultados; caso contrário, considera-se a mediana.

Vale salientar que quando a diferença absoluta excede " $r$ " significa que a diferença é considerada suspeita e recomenda-se investigar a causa dos resultados suspeitos. Procedimento similar é conduzido para avaliar diferenças entre dois (ou mais) resultados interlaboratoriais com o índice da reprodutibilidade (R).

Observa-se que os índices r e R são dinâmicos e devem ser reavaliados com certa frequência, através de nova rodada dos trabalhos de comparação interlaboratorial. ${ }^{10}$

\section{TESTE DE REJEIÇÃO DOS VALORES DISCREPANTES - TESTE DE COCHRAN E TESTE DE GRUBBS}

O teste de Cochran, para verificação de variâncias, é aplicado para amostras de mesmo tamanho, ou seja, quando os laboratórios obtêm resultados com o mesmo número de replicatas, o que acontece 
geralmente em programas interlaboratoriais. Ë um teste unilateral que verifica o maior valor, quando se relaciona a variância mais alta com

a soma total das variâncias.
A hipótese nula é verificada pela estatística $C=\frac{\max s_{i}^{2}}{\sum s_{i}^{2}}$, sendo

$C$ denominado coeficiente de Cochran e $i=1,2, \ldots ., \mathrm{k}$. Compara-se o valor calculado $C$ com os valores críticos tabelados $C_{c}$ em teste de 1 ou $5 \%$ de significância. ${ }^{11,13,15} \mathrm{~A}$ decisão a ser tomada segue o seguinte critério:

- se $C<C_{c}$ (no nível de significância de 5\%)aceita-se a hipótese nula

- se $C_{c}(1 \%)>C>C_{c}(5 \%)$

- se $C>C_{c}(1 \%)$ situação suspeita rejeita-se a hipótese nula

Excluídos os laboratórios pelas variâncias discrepantes (se houver), segue-se ao teste de Grubbs, que verifica discrepância pelas médias.

$\mathrm{O}$ teste de Grubbs verifica preliminarmente a existência de um valor anômalo em cada extremidade do conjunto. Se nesta primeira análise um dos dois valores for considerado discrepante, ele é excluído do conjunto e novo teste, verificando o valor seguinte em cada extremidade do conjunto, é realizado e assim sucessivamente; caso contrário, se nesta primeira análise, ambos os valores forem aceitos como não discrepantes, o teste é então realizado verificando-se dois outros valores em cada extremidade do conjunto. Se nesta segunda análise os dois resultados de uma das extremidades forem considerados discrepantes, eles deverão ser excluídos do conjunto e novo teste, verificando os dois valores seguintes em cada extremidade do conjunto, é realizado e assim sucessivamente.

$\mathrm{O}$ valor mais alto e o valor mais baixo do conjunto de resultados são comparados com o valor médio $\bar{x}$ do conjunto dividido pelo desvio padrão $s$ do conjunto. Considerando-se a análise de valores $\mathrm{x}_{\mathrm{i}}(\mathrm{i}=1$ a $n$ ) obtém-se para o valor mais baixo, a estatística

$G_{1}=\frac{x-x_{1}}{s}$

e, para o valor mais alto,

$G_{n}=\frac{x_{n}-x}{s}$

Os resultados para $G_{i}(\mathrm{i}=1,2, \ldots n)$ são comparados com o valor da estatística $G_{c}$, para testes com nível de significância de 1 e $5 \%$, obtidos de tabelas. ${ }^{13,15}$ Seguindo o critério:

- se $G_{i} \leq G_{c}(5 \%)$

- se $G_{c}(1 \%) \geq G_{i} \geq G_{c}(5 \%)$

- se $G_{i}>G_{c}(1 \%)$

a hipótese nula é aceita valor é suspeito

Se a hipótese nula é aceita, passa-se à segunda etapa do teste de Grubbs: calcula-se a soma dos quadrados das diferenças em relação à sua média $\left(\mathrm{SQ}_{\mathrm{t}}\right)$ considerando todos os resultados e a soma dos quadrados das diferenças em relação à nova média excluídos os dois menores valores $\left(\mathrm{SQ}_{1,2}\right)$. Calcula-se a estatística $G^{\prime}=\mathrm{SQ}_{1,2} /$ $\mathrm{SQ}_{\mathrm{t}}$ e se resultar em valor maior do que o valor de $G_{c}$ tabelado para testes de 1 e 5\% de significância, aceita-se a hipótese nula. Salientase, aqui, que o critério de aceitação é o contrário do anterior. Se $G$ for menor ou igual aos valores críticos tabelados, exclue-se o mais baixo e repete-se o procedimento com os dois menores seguintes. Em seguida, desconsiderando-se os dois maiores valores, repete-se o procedimento. Considerando "p-1" e "p", respectivamente, os dois valores mais altos, calcula-se $\mathrm{SQ}_{\mathrm{p}-1, \mathrm{p}}=$ a soma dos quadrados das diferenças excluídos os dois valores mais altos. $\mathrm{Se} G^{\prime \prime}=\mathrm{SQ}_{\mathrm{p}-1, \mathrm{p}} / \mathrm{SQ}_{\mathrm{t}}$ for maior que os valores de $G_{c}$ tabelados (no nível de significância
1 e 5\%) a hipótese nula é aceita. Caso contrário, com $G^{\prime \prime}$ menor ou igual aos valores críticos tabelados, exclue-se o mais alto e repete-se o procedimento considerando os dois maiores seguintes. O critério de aceitação/rejeição é descrito a seguir:
- se $G^{\prime}\left(\right.$ ou $\left.G^{\prime \prime}\right) \leq G_{c}(5 \%)$

- se $G_{c}(1 \%) \geq G^{\prime}\left(\right.$ ou $\left.G^{\prime \prime}\right) \geq G_{c}(5 \%)$

- se $G^{\prime}\left(\right.$ ou $\left.G^{\prime \prime}\right)>G_{c}(1 \%)$ valor considerado anômalo valor é suspeito aceita-se a hipótese nula
Há uma recomendação para que o número de valores considerados discrepantes não ultrapasse de $10 \% .{ }^{10}$ Acima de $15 \%$ todo o conjunto de dados deve ser desconsiderado. Se o número de anômalos estiver entre 10 a 15\%, o responsável pela avaliação dos dados deverá decidir se continua os cálculos com a análise de variância.

\section{OBJETIVO}

Este trabalho tem o objetivo de divulgar o uso dos índices de repetitividade e de reprodutibilidade e conscientizar os laboratórios dos benefícios que os mesmos podem usufruir com a participação em programas interlaboratoriais.

\section{METODOLOGIA}

Vários laboratórios industriais de controle de qualidade de material silício metálico grau químico manifestaram interesse em ter acesso a um padrão analítico para o monitoramento da qualidade de seus resultados. Assim, como etapa preliminar, foi planejado um programa interlaboratorial para a determinação dos valores de índices r e R, em estudo de precisão de método de ensaio para a determinação de ferro total em amostras de silício metálico. Na ocasião, doze laboratórios colaboraram nesse estudo, empregando a técnica da espectrometria de absorção atômica com chama, por ser a técnica analítica mais comumente empregada. Os laboratórios participantes eram laboratórios envolvidos no controle de qualidade de silício metálico grau químico, laboratórios de institutos de pesquisas e de prestação de serviços. O material foi especialmente preparado para este fim no Laboratório de Materiais de Referência Inorgânicos do Instituto de Pesquisas Tecnológicas do Estado de São Paulo S.A., sendo distribuídas amostras com a homogeneidade comprovada e material com granulometria passante em peneira de abertura de malha de $0,152 \mathrm{~mm}$. Cada laboratório recebeu uma amostra de $50 \mathrm{~g}$ e efetuou seis replicatas. Com os dados brutos, foram aplicados os testes de Cochran e de Grubbs para verificar se havia valores discrepantes, antes de iniciar o cálculo para a análise de variância - fator único (laboratórios) e obtenção dos índices r e R. Em seguida, um estudo de caso foi ilustrado para exemplificar como aceitar ou rejeitar um resultado analítico gerado em trabalho de rotina pós-interlaboratorial, considerando os índices $\mathrm{r}$ e R obtidos.

\section{RESULTADOS E DISCUSSÃO}

A Tabela 2 mostra os resultados, expressos em \% de Fe total, obtidos por 12 laboratórios em comparação interlaboratorial.

Para verificar a homocedasticidade das variâncias foi aplicado o teste de Cochran. Preliminarmente, foram calculadas as médias e as variâncias para cada conjunto de resultados dos laboratórios.

Com os resultados dos 12 laboratórios e pelo critério de Cochran, verificou-se que o laboratório 4 apresentava a variância mais alta. Em seguida, calculou-se o valor da estatística $C$ para o mesmo, denominando-a de $C_{4}$, sendo que o valor obtido $\left(C_{4}=1,77.10^{-4} / 41,83 \cdot 10^{-5}=\right.$ $0,4231)$ mostrou-se maior do que os valores críticos tabelados ${ }^{15} \mathrm{em}$ testes de $5 \%\left(C_{c}=0,2634\right)$ e $1 \%\left(C_{c}=0,3099\right)$. Assim, o laboratório 
4 foi excluído como laboratório apresentando resultados discrepantes dos demais do conjunto. Em sequência, a variância seguinte mais alta indicada como a do laboratório 11 foi testada, usando o mesmo critério, obtendo-se $C_{11}=9,19 \cdot 10^{-5} / 2,41 \cdot 10^{-4}=0,3813$. Sendo $C_{11}$ $>C_{c}(0,2810)$ em teste de $5 \%$ de significância, o laboratório 11 também foi excluído. Testando o laboratório 8 , verificou-se que $C_{8}$ $=2,83 \cdot 10^{-5} / 1,49 \cdot 10^{-4}=0,1899$; e sendo $C_{8}$ menor que os respectivos valores tabelados no nível de $5 \%\left(C_{c}=0,3029\right)$ e $1 \%\left(C_{c}=0,3572\right)$ manteve-se o laboratório 8 . Verificadas as variâncias, passou-se ao teste de Grubbs, para verificação de compatibilidade de médias, classificando os laboratórios por ordem crescente de seus valores. $\mathrm{E}$ usando as Equações 5 e 6, $\operatorname{com} \bar{x}=0.28246, x_{1}=0.2467, x_{n}=0.3067$ e $s=0,016768$ obteve-se $G_{1}=2,1326$ e $G_{n}=1,4456$, ambos menores que os valores críticos tabelados ${ }^{15}, G_{c}(5 \%)=2,290$ e $G_{c}(1 \%)=2,482$. Com a hipótese nula aceita, a segunda etapa do teste de Grubbs foi aplicada, testando os dois maiores e os dois menores resultados.

Tabela 2. Resultados, em \% Fe total, obtidos em programa interboratorial

\begin{tabular}{|c|c|c|c|c|c|c|c|}
\hline lab & & & repli & catas & & média & variância \\
\hline 1 & 0,270 & 0,280 & 0,280 & 0,270 & 0,280 & $0,2800,2766$ & 0,0000267 \\
\hline 2 & 0,301 & 0,300 & 0,302 & 0,304 & 0,304 & $0,3010,3020$ & 0,0000028 \\
\hline 3 & 0,285 & 0,288 & 0,295 & 0,282 & 0,290 & $0,2880,2880$ & 0,0000196 \\
\hline 4 & 0,292 & 0,279 & 0,287 & 0,316 & 0,281 & $0,2910,2910$ & 0,0001772 \\
\hline 5 & 0,276 & 0,270 & 0,281 & 0,270 & 0,280 & $0,277 \quad 0,2757$ & 0,0000227 \\
\hline 6 & 0,282 & 0,284 & 0,285 & 0,287 & 0,286 & $0,2850,2848$ & 0,0000030 \\
\hline 7 & 0,277 & 0,279 & 0,280 & 0,273 & 0,278 & $0,280 \quad 0,2778$ & 0,0000070 \\
\hline 8 & 0,246 & 0,256 & 0,249 & 0,241 & 0,245 & $0,2430,2467$ & 0,0000283 \\
\hline 9 & 0,280 & 0,278 & 0,284 & 0,281 & 0,275 & $0,2770,2792$ & 0,0000102 \\
\hline 10 & 0,300 & 0,307 & 0,311 & 0,309 & 0,307 & $0,3060,3067$ & 0,0000139 \\
\hline 11 & 0,301 & 0,290 & 0,284 & 0,308 & 0,293 & $0,2840,2933$ & 0,0000919 \\
\hline 12 & 0,272 & 0,277 & 0,269 & 0,270 & 0,278 & $0,270 \quad 0,2727$ & 0,0000151 \\
\hline
\end{tabular}

Excluindo os dois menores, a média resultou em 0,28815 e sem os dois maiores, a média foi de 0,27699 . Com $\mathrm{SQ}_{\mathrm{t}}=0,80037, \mathrm{SQ}_{1,2}$ $=0,66514$ e $\mathrm{SQ}_{9,10}=0,61510$, obteve-se $G^{\prime}=0,66514 / 0,80037=$ 0,8310 e $G^{\prime \prime}=0,61510 / 0,80037=0,7685$. Sendo $G_{c}(5 \%)=0,1864$ e $G_{c}(1 \%)=0,1150$, concluiu-se que $G^{\prime}$ e $G^{\prime \prime}$ atendem o critério para aceitação das médias.

Como resultado final, após a aplicação dos testes de Cochran e de Grubbs, considerou-se a exclusão dos resultados dos laboratórios 4 e 11. Dois laboratórios excluídos do total de 12 representam $17 \%$ de discrepantes, o que não é desejável, conforme recomendação citada anteriormente. A rigor, novo experimento deveria ser conduzido para a obtenção dos índices desejados. Porém, foi decidido continuar com a análise de variância, calculando-se valores preliminares que servirão de indicadores para avaliar o desempenho dos laboratórios envolvidos em rodadas posteriores. Sendo assim, procedeu-se à análise de variância.

A Tabela 3 apresenta o resumo da análise de variância - fator único indicando a decomposição da variância total representada pelo conjunto dos dados de 10 laboratórios.

A variância total devido aos 10 laboratórios compreende a soma das variâncias devido à variância dentro dos respectivos laboratórios e à combinação das variâncias devido a laboratórios e interação amostra/laboratórios. Usando as Equações 2, 3 e 4 e considerando que:
$Q M_{\text {entre }}=$ variância total $=0,001648 ; s_{\text {dentro }}^{2}$, correspondendo a $Q M_{\text {dentro }}=1,49 \times 10^{-5} \mathrm{e} \mathrm{s}^{2}{ }_{\text {entre }}$, correspondendo à variância entre laboratórios e interação amostra/laboratório foram calculados os valores para os índices de repê (r) e Reprô (R).

$$
s_{\text {entre }}^{2}=\frac{0,001648-0,0000149}{6}=0,00027218
$$

$r($ repê $)=2,8 \sqrt{1,49 \times 10^{-5}}=0,011$

$$
\mathrm{R}(\text { reprô })=2,8 \sqrt{0,0000149+0,00027218}=0,047
$$

O valor da Reprô inclui, em seu cálculo, o valor da repê. Portanto, Reprô é necessariamente maior que repê. Observa-se que, para a obtenção de valores mais baixos, é necessário reduzir as variabilidades dentro de laboratórios, bem como é desejável uma harmonização dos procedimentos tal que as variabilidades entre laboratórios (devido a diferentes equipamentos, recursos humanos e meio ambientes) possam ser minimizadas.

Tabela 3. Resumo da análise de variância - fator único (laboratórios) excluídos os resultados dos laboratórios 4 e 11

\begin{tabular}{lccc}
\hline $\begin{array}{l}\text { Fontes de } \\
\text { Variação }\end{array}$ & $\begin{array}{c}\text { Soma de } \\
\text { Quadrados }\end{array}$ & $\begin{array}{c}\text { Graus de } \\
\text { liberdade }\end{array}$ & $\begin{array}{c}\text { Quadrados } \\
\text { Médios }\end{array}$ \\
\hline entre lab & 0.014834 & 9 & 0.001648 \\
dentro lab & 0.000745 & 50 & $1.49 \mathrm{E}-05$ \\
Total & 0.015579 & 59 & \\
\hline
\end{tabular}

Em seguida são apresentados três casos de aplicação dos índices de repetitividade $(r=0,011)$ e de reprodutibilidade $(R=0,047)$, relacionando-os à precisão de resultados de medições em atividade de rotina para o controle interno da qualidade (situação pós-programa interlaboratorial):

CASO 1: um laboratório obtém 2 replicatas: 0,273 e 0,279\%; a diferença de $0,006 \%$ entre as duas replicatas é aceitável, pois $\mathrm{r}=0,011$; portanto os dois resultados são válidos.

CASO 2: um laboratório obtém 2 replicatas: 0,299 e 0,316\%; a diferença de $0,017 \%$ entre as duas replicatas é inaceitável $(\mathrm{r}=0,011)$; um dos valores deve ser rejeitado.

CASO 3: dois laboratórios: um laboratório obtém $0,269 \%$ e o outro $0,299 \%$; a diferença de $0,030 \%$ entre os dois resultados é menor que o valor de R $(0,047)$; portanto, os dois valores são aceitos.

Os valores obtidos para $\mathrm{r}$ e $\mathrm{R}$, no estudo de caso, indicam que eles são abertos, tolerando variações em faixa ampla de valores. O resultado final sugere uma incerteza apreciável. No exemplo, após a exclusão de 2 dos 12 laboratórios participantes, foram considerados 10 laboratórios, 1 amostra e 6 replicatas, ou seja, [(10 x 1 x 6) - 1] variáveis independentes ou 59 graus de liberdade, valor bastante elevado, para um programa ainda simples. Valores elevados de $\mathrm{r}$ e $\mathrm{R}$ indicam existência de problemas. É metrologicamente desejável obter índices mais fechados, reduzindo-se os valores de repê e Reprô. Para isso, é necessário envidar esforços para reduzir as variabilidades dentro dos laboratórios. No entanto, apesar de abertos, podem ser inicialmente utilizados como indicadores para, em etapa posterior, obter a redução dos mesmos. A diminuição desses valores indica melhoria na qualidade das medições.

O diagrama de causa e efeito ou diagrama de Ishikawa, também conhecido como espinha de peixe, é uma ferramenta que permite estruturar hierarquicamente as causas de determinado problema e auxiliar que as ações corretivas sejam tomadas. ${ }^{18}$ Sua aplicação é muito recomendada para identificar problemas em processos de medição. 
Laboratórios que efetivamente podem fazer uso, na rotina, dos índices de repê e Reprô, são apenas os participantes do programa interlaboratorial planejado para esse fim. Outros laboratórios podem recorrer a esses índices como valores informativos.

Laboratórios participantes de programas interlaboratoriais devem ser atuantes na área e os ensaios de interesse devem fazer parte de sua rotina de trabalho. É recomendável que haja uma participação constante dos mesmos laboratórios inscritos no programa, a fim de que possa ser alcançado um alto grau de conscientização e de disciplina nas atividades. Os benefícios advindos dos esforços, que grupos de laboratórios se propõem a dedicar em conjunto, favorecem o trabalho para o estabelecimento de normas técnicas (para métodos de ensaios e especificações), o aprimoramento da qualidade laboratorial e até a redução de custos de calibração de instrumentos, apontando a necessidade de ajustes e/ou manutenção.

\section{CONCLUSÃO}

Valores de repê e Reprô têm ampla aplicação metrológica e industrial, especialmente no esclarecimento de controvérsias, tipo fornecedor-usuário, ou seja, dois laboratórios. Esses dois laboratórios podem ser da mesma organização ou de empresas diferentes. Participando de programas interlaboratoriais e utilizando os índices de repê e de Reprô, os laboratórios usufruem de um mecanismo para garantir a estabilidade de seus processos de medição.

O procedimento para a obtenção desses índices é bastante simples. Não requer cálculos complicados. As ferramentas de análise de dados encontradas no software do Excel são fáceis de usar e suficientes para os cálculos propostos. Experimentos desse tipo podem ser adotados por empresas que precisam manter sob controle a qualidade analítica de seus vários laboratórios sediados em regiões distantes entre si.

É importante lembrar que os valores de $\mathrm{r}$ e R são múltiplos de desvio padrão específicos, sendo assim associados à precisão. Não estão associados à exatidão e, portanto, não são suficientes para garantir a rastreabilidade dos resultados de medição.

Para o cálculo de r e R supõe-se que entre as variabilidades dos laboratórios existam somente diferenças muito pequenas. Experiências, entretanto, mostram que este nem sempre é o caso, de modo que um teste estatístico deve ser incluído para confirmar a validade desta suposição.

\section{AGRADECIMENTOS}

À FINEP e CNPq pelos recursos para auxílio à pesquisa e bolsas DTI concedidas; e ao Laboratório de Materiais de Referência Inorgânicos do Instituto de Pesquisas Tecnológicas do Estado de São Paulo S.A., onde o material silício metálico foi preparado.

\section{REFERÊNCIAS}

1. Detaille, R.; Maetz, P.; J. Accred. Qual. Assur. 2006, 11, 8 .

2. NBR ISO/IEC 17025:2005, Requisitos gerais para competência de laboratórios de ensaios e calibração, Associação Brasileira de Normas Técnicas, Rio de Janeiro, 2005.
3. NBR ISO/IEC Guia 43:1999, Ensaios de proficiência por comparações interlaboratoriais. Parte 1: Desenvolvimento e operação de programas de ensaios de proficiência, Associação Brasileira de Normas Técnicas, Rio de Janeiro, 1999.

4. Chui, Q. S. H.; Antonoff, H. B.; Olivieri, J. C.; Quim. Nova 2002, 25, 657.

5. Chui, Q. S. H.; Bispo, J. M. A.; Iamashita, C. O.; Quim. Nova 2004, 27, 993.

6. ISO 5725-1:1994, Accuracy (trueness and precision) of Measurement Methods and Results. Part 1: General principles and definitions. Statistical methods for quality control, International Organization for Standardization, Geneva, 1994.

7. ISO DIS 13528:2002, Statistical methods for use in proficiency testing by interlaboratory comparisons, International Organization for Standardization, Geneva, 2002.

8. INMETRO DOQ-CGCRE-008:2007, Revisão 2. Orientação sobre validação de métodos de ensaios químicos, Instituto Nacional de Metrologia, Normalização e Qualidade Industrial, Rio de Janeiro, 2007.

9. ABNT NBR 14597:2000, Precisão de Métodos Analíticos Determinação da Repetitividade e Reprodutibilidade de Métodos para Ensaios de Produtos Químicos - Estudo Intralaboratorial, Associação Brasileira de Normas Técnicas, Rio de Janeiro, 2001.

10. Waeny, J. C. C.; Associação Brasileira de Controle da Qualidade(ABCQ)- Nota Técnica 17/90 Repetitividade e Reprodutividade III, São Paulo, 1990.

11. Farrant, T.; Practical Statistics For The Analytical Scientist - A Bench Guide, LGC/RSC, UK, 1997.

12. Bussab, W. de O.; Morettin, P. A.; Estatística Básica, Ed. Saraiva, 5ª ed., 2007.

13. Costa Neto, P. L. de O.; Estatística, Ed. Edgard Blücher Ltda, $12^{\mathrm{a}}$ reimpressão, 1992.

14. ASTM E 178:2002, Standard Practice for Dealing with Outlying Observations, American Society for Testing and Materials, USA, 2002.

15. ISO 5725-2:1994, Accuracy (trueness and precision) of Measurement Methods and Results. Part 3: Basic method for the determination of repeatability and reproducibility of a standard measurement method, International Organization for Standardization, Geneva, 1998.

16. Campos, P. de M.; Muniz, J. A.; Oliveira, M. S. de; Ferreira, D. F.; Ciência e Agrotecnologia 1999, 23, 404.

17. ISO 5725-6:1994, Accuracy (trueness and precision) of Measurement Methods and Results. Part 6: Use in practice of accuracy values, International Organization for Standardization, Geneva, 2001.

18. Taylor, K. T.; Quality Assurance of Chemical Measurements, Lewis Publishers, INC., 1987; Juran, J. M.; Gryna, F. M.; Juran's Quality Control Handbook, $4^{\text {th }}$ ed., 1988, McGraw Hill: New York; EURACHEM/ CITAC/AOAC International Guide, Quantifying Uncertainty in Analytical Measurement, $2^{\text {nd }}$ ed., 1999, Draft: EURACHEM Workshop, Helsinki; Christensen, E. H.; Coombes-Betz, M.; Marilyn, S.; The Certified Quality Process Analyst Handbook, ASQ Quality Press Publisher, 2007; Reyes, A. E. L.; Vicino, S. R.; Qualidade Total: Diagrama de Ishikawa, http//www.esalq.usp.br/qualidade/ishikawa/pag1.htm., acessada em Fevereiro 2009. 